\title{
Obstruction of right ventricular outflow tract by solitary ovarian metastasis
}

\author{
D. N. W. GRIFFITH AND A. MYERS \\ From the Department of Cardiology, Royal Free Hospital, Pond Street, London
}

SUMMARY We report a case in whom there was right ventricular outflow tract obstruction by a solitary metastatic deposit from an ovarian primary. The patient presented with signs of right ventricular overload and the outflow tract obstruction was recognised at cardiac catheterisation, but the final diagnosis was only revealed at necropsy.

Right ventricular outflow tract obstruction by tumours of any type is rare, the commonest lesions involved being primary cardiac tumours, particularly fibrosarcoma and rhabdomyoma. Obstruction by secondary carcinoma is even less common, only 2 cases having been reported; in one there was a bronchogenic primary (McLoughlin, 1970), and in the other a primary renal carcinoma (Gordon et al., 1973). In the following report we describe what we believe to be the first case of right ventricular outflow tract obstruction by a metastatic deposit from an ovarian carcinoma.

\section{Case report}

A 64-year-old woman presented with a history of the sudden onset of breathlessness four months previously. She continued to be breathless on exertion and when first seen in hospital it was noted that she was dyspnoeic on walking up only two or three stairs. She had had no chest pain. She had lost her appetite and had lost $6.35 \mathrm{~kg}(14 \mathrm{lb})$ in the past two months. She had smoked 20 cigarettes a day for many years until six months previously. On examination she was a fit-looking 64-year-old woman, who was not distressed or dyspnoeic at rest. The pulse was normal and blood pressure was $160 / 100 \mathrm{mmHg}$. Jugular venous pressure was normal. On auscultation a loud pulmonary component of the second sound and a loud fourth sound were heard, and 4 days after admission a short mid-systolic murmur at the pulmonary area and a short pulmonary early diastolic murmur appeared. The only other abnormality was a smooth, non-tender enlargement of the liver to $2 \mathrm{~cm}$ below the costal margin.

The initial clinical diagnosis was recurrent pulmonary emboli. Chest $x$-ray, lung scan, and echocardiogram were normal. The electrocardiogram showed right atrial overload and right ventricular hypertrophy with partial right bundlebranch block and QRS axis $+120^{\circ}$ (Fig. 1). The electrocardiogram recorded 2 years before was completely normal. Other routine tests gave normal results.

At cardiac catheterisation the pulmonary artery was entered with difficulty, and on withdrawal a gradient of $42 \mathrm{mmHg}$ across the right ventricular outflow tract was recorded (Fig. 2). Right ventricular pressure was $58 / 0 \mathrm{mmHg}$ and main pulmonary artery pressure $16 / 3 \mathrm{mmHg}$. A large $a$ wave was recorded in the right atrium with $a 20, x-1$, $v 7, y 4$, and mean pressure $7 \mathrm{mmHg}$. A test injection of contrast medium produced a severe reaction, with vomiting and hypotension, and the

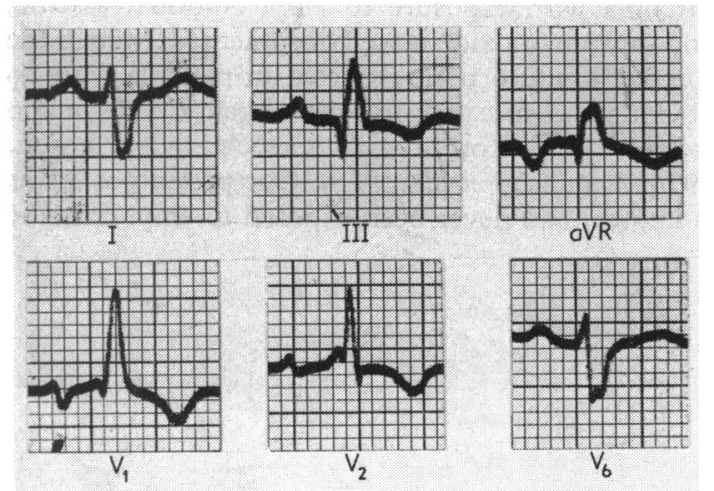

Fig. 1 Electrocardiogram at time of presentation. 
procedure was ended without right ventriculography.

Subsequent urinary 5-hydroxyindole-acetic acid estimation was normal. Liver scan was interpreted as showing multiple space-occupying lesions, but a liver biopsy failed to show evidence of tumour. During this phase of her illness she developed intermittent left cerebellar signs, but a brain scan was negative and an echoencephalogram showed no midline shift. An electroencephalogram showed only diffuse abnormality.

During this period of investigation, the patient's condition had progressively deteriorated. She latterly appeared cachectic and eventually died five weeks after admission.

\section{NECROPSY}

The heart weighed $540 \mathrm{~g}$. There was a pericardial effusion. The right ventricle showed extensive tumour deposits filling much of the cavity and involving the cusps of the tricuspid valve (Fig. 3). The pulmonary valve was normal. Thrombus was found in the right atrial appendage and the right atrium was dilated. A mass of tumour was found in the right ovary which was bound to the pelvic wall. No tumour deposits were found in the brain or liver or any other organ.

Histology of the ovary and heart showed undifferentiated carcinoma of identical appearance in the two sites, and suggested an ovarian primary with a solitary myocardial metastasis.

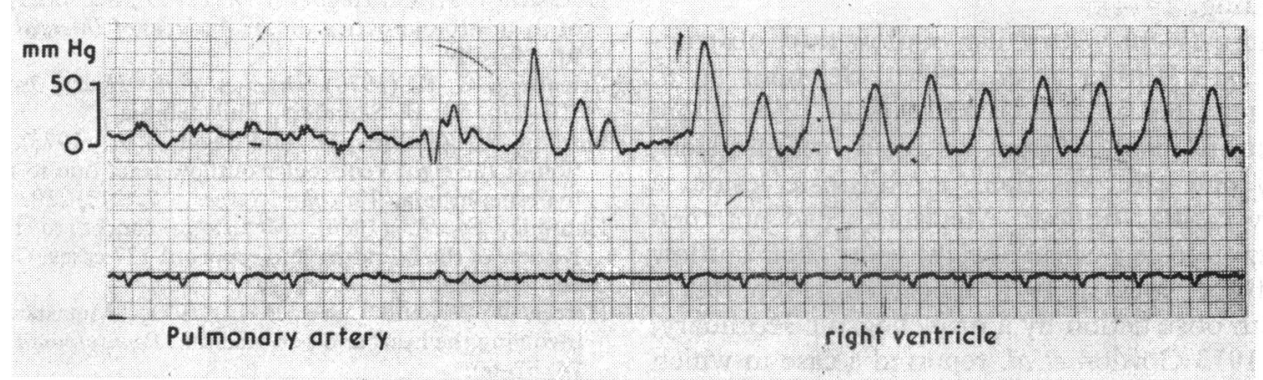

Fig. 2 Withdrawal pressure record from pulmonary artery to right ventricle.

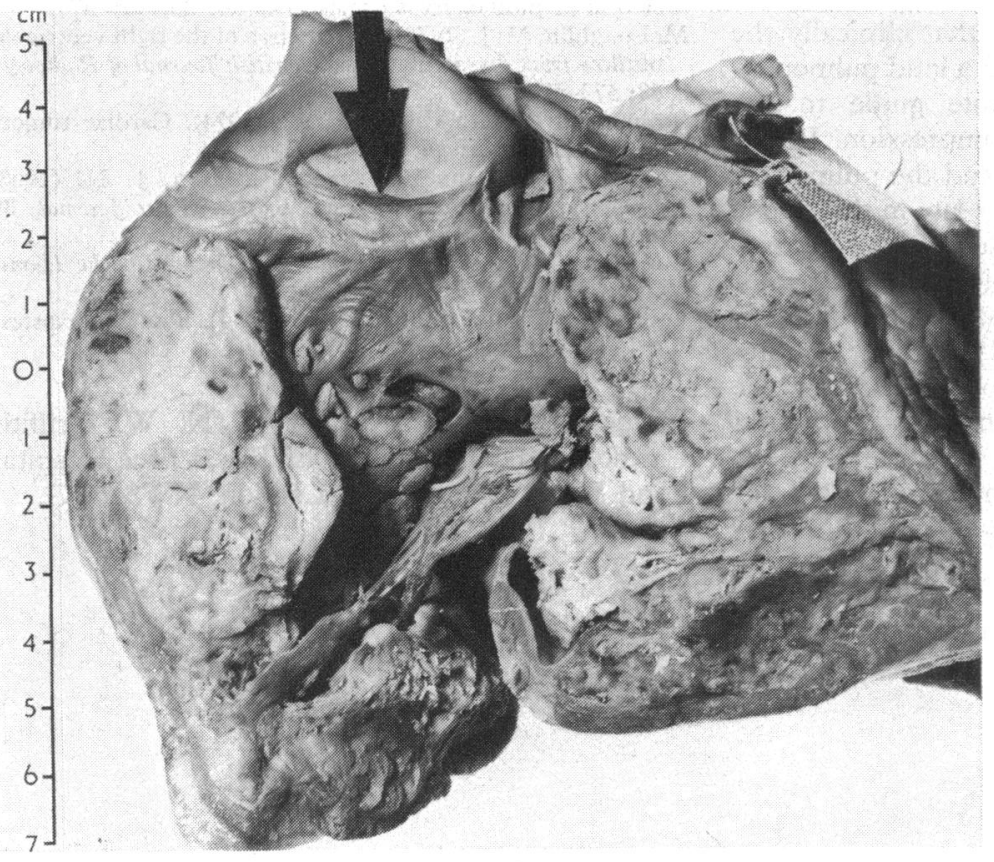

Fig. 3 Photograph of interior of right ventricle. 


\section{Discussion}

The incidence of cardiac involvement by tumours has been reported as 0.05 per cent for primary tumours and 0.25 per cent to 1 per cent for secondary tumours (Friedberg, 1970). However, in patients with known carcinoma the incidence of cardiac metastases is higher. Willis (1934) reported an incidence of 6.2 per cent of myocardial metastases in 323 necropsy cases. With inclusion of all cardiac metastases the incidence rises further and has been reported as 13.9 per cent (De Loach and Haynes, 1953), 19.1 per cent (Young and Goldman, 1954), and 17.3 per cent (Biran et al., 1969). The commonest tumours giving rise to cardiac metastases are lung and breast carcinoma, melanoma, and leukaemias (Harrer and Lewis, 1971; Scobbo and Chung, 1974).

Acquired right ventricular outflow tract obstruction is rare (Littler et al., 1970). Seymour et al. (1968) reported a series of 13 cases in 12 of whom obstruction resulted from extrinsic compression; in only one case was there an intrinsic lesion, a primary cardiac tumour. Metastases had not then been reported as a cause of right ventricular outflow tract obstruction. In $1970 \mathrm{McLoughlin}$ reported a case with obstruction by a bronchogenic secondary, and in 1973 Gordon et al. reported a case in which the cause was a secondary from a renal carcinoma. This was diagnosed by catheterisation and at operation the tumour was excised from the wall of the right ventricle.

Littler et al. (1970) suggested that clinically the triad of chest pain, dyspnoea, and a loud pulmonary systolic murmur was a valuable guide to the diagnosis of pulmonary artery compression. In our patient there was no chest pain and the pulmonary systolic murmur was only evident late in the course of the disease and was never loud. The electrocardiogram may be a valuable diagnostic aid. Hanfling (1960) observed that the septum was often involved in cardiac tumours and that right bundlebranch block was relatively common. Our case showed right ventricular hypertrophy and right bundle-branch block. Harris et al. (1965) suggested that arrhythmias were by far the most frequent abnormality in patients with $\stackrel{\frac{\rho}{c}}{c}$ cardiac metastases, but in our patient no arrhythmia was observed. Cardiac metastasis was not $\stackrel{\vec{\sim}}{\rightarrow}$ suspected though carcinomatosis was, despite our inability to establish this during life. The cerebellar signs were presumably a non-metastatic mani- $\frac{N}{\vec{D}}$ festation of the ovarian carcinoma, and false $\stackrel{\varnothing}{\unrhd}$ positive liver scans are well known.

We are grateful to Dr C. Symons for allowing us to publish details of a case under his care.

\section{References}

Biran, S., Hochman, A Levij, I S and Stern, S. (1969) Clinical diagnosis of secondary tumors of the heart and $\bigcirc$ pericardium. Diseases of the Chest, 55, 202-208.

DeLoach, J. F., and Haynes, J. W. (1953). Secondary tumours of the heart and pericardium. Archives of Internal Medicine, 91, 224-249.

Friedberg, C. K. (1970). Diseases of the Heart, 3rd ed., pp. 707-720. W. B. Saunders, Philadelphia.

Gordon, R., Kimbiris, D., and Segal, B. L. (1973). Obstruc- $\overrightarrow{0}$ tion of the right ventricular outflow tract due to metastatic $\infty$ hypernephroma. Vascular Surgery, 7, 213-219.

Hanfling, S. M. (1960). Metastatic cancer to the heart. Review of the literature and report of 127 cases. Circulation, 22, 474-483.

Harrer, W. V., and Lewis, P. L. (1971). Metastatic tumors involving the heart and pericardium. Pennsylvania Medicine, $\frac{\mathrm{D}}{\mathrm{Q}}$ 74, 57-60.

Harris, T. R., Copeland, G. D., and Brody, D. A. (1965). $\overrightarrow{\bar{O}}$ Progressive injury current with metastolic tumor of the heart. American Heart fournal, 69, 392-400.

Littler, W. A., Meade, J. B., and Hamilton, D. I. (1970). Acquired pulmonary stenosis. Thorax, 25, 465-471.

McLoughlin, M. J. (1970). Obstruction of the right ventricular outflow tract due to metastasis. British fournal of Radiology, 43, 573-576.

Scobbo, R. R., and Chung, E. K. (1974). Cardiac tumors. Postgraduate Medicine, 56, 138-141.

Seymour, J., Emanuel, R., and Pattinson, J. N. (1968). Acquired pulmonary stenosis. British Heart fournal, 30, 776-785.

Willis, R. A. (1934). The Spread of Tumours in the Human 음 Body. J. and A. Churchill, London.

Young, J. J., and Goldman, I. R. (1954). Tumor metastasis to the heart. Circulation, 8, 220-229.

Requests for reprints to Dr D. N. W. Griffith, N Cardiology Department, The Royal Free Hospital, N Pond Street, Hampstead, London, NW3 2QG. 